\title{
Prenatal diagnosis of congenital high airway obstruction syndrome
}

\author{
Víctor Lago Leal, Luis Martínez Cortés, Carlos Seco Del Cacho \\ Department of Obstetrics and Gynecology, University Hospital of Getafe, Getafe, Madrid, Spain
}

Correspondence: Dr. Víctor Lago Leal, Department of Obstetrics and Gynecology, University Hospital of Getafe, Getafe, Address: Carr. Madrid - Toledo, Km 12,500, 28905 Getafe, Madrid, Spain. E-mail: victor.lago.leal@hotmail.com

\begin{abstract}
Congenital high airway obstruction syndrome (CHAOS) is a rare sequence due to a complete obstruction of the fetal airway that blocks the larynx or trachea, either intrinsic atresia or extrinsic compression (e.g., congenital neck mass). Despite the true incidence of CHAOS is unknown, an incidence of 1 per 50,000 newborns is described. If any obstruction occurs in the tracheobronchial airway, this secretion cannot be extricated. Because of this situation, a knock-on effect starts: the enlargement of the lungs squeezes the heart and great veins, what results in a replacement of the heart to the center of the chest becoming small and dysfunctional. Decreased venous return and the failure of cardiovascular system end in ascites and hydrops. The diaphragm planes or inverts according to the severity of the process. In case of unrecognized syndrome during the prenatal period, it usually results in stillbirth or death shortly after delivery. The overall prognosis remains fatal, and long-term medical and surgical challenges for survivors remain considerable even after overcoming fetal intervention.
\end{abstract}

Key words: Congenital high airway obstruction syndrome; magnetic resonance imaging; newborn; tracheal agenesis; ultrasound

\section{Introduction}

Congenital high airway obstruction syndrome (CHAOS) is a rare sequence due to a complete obstruction of the fetal airway that blocks the larynx or trachea, either intrinsic atresia or extrinsic compression (e.g., congenital neck mass). ${ }^{[1]}$ Despite the true incidence of CHAOS remains unknown, an incidence of 1 per 50,000 newborns is described. ${ }^{[2]}$

The more common pathogenetic theory for this lesion involves a decreased blood supply during the embryogenetic period, what may affect the normal development of the trachea/larynx.

\begin{tabular}{|l|l|}
\hline \multicolumn{2}{|c|}{ Videos available on: www.ijri.org } \\
\hline \multicolumn{2}{|c|}{ Access this article online } \\
\hline Quick Response Code: & Website: \\
& www.ijri.org \\
\hline
\end{tabular}

In a normal evolution, through the tracheobronchial tree, the fluid secreted by fetal lung is absorbed. Nevertheless, if any obstruction occurs in the tracheobronchial airway, this secretion cannot be extricated. Because of this situation, the lungs' pressure steadily raises conditioning to an enlargement of the lungs. A knock-on effect starts: the enlargement of the lungs squeezes the heart and great veins, what results in a replacement of the heart to the center of the chest becoming small and dysfunctional. Decreased venous return and the failure of cardiovascular system end in ascites and hydrops. The diaphragm planes or inverts according to the severity of the process. ${ }^{[3]}$

Due to advances in imaging techniques, the syndrome can be recognized prenatally. Since the initial report of its prenatal diagnosis in 1989 by Arizawa et al., ${ }^{[4]}$ more

This is an open access journal, and articles are distributed under the terms of the Creative Commons Attribution-NonCommercial-ShareAlike 4.0 License, which allows others to remix, tweak, and build upon the work non-commercially, as long as appropriate credit is given and the new creations are licensed under the identical terms.

For reprints contact: reprints@medknow.com

Cite this article as: Lago Leal V, Martínez Cortés L, Seco Del Cacho C. Prenatal diagnosis of congenital high airway obstruction syndrome. Indian J Radiol Imaging 2018;28:366-8. 


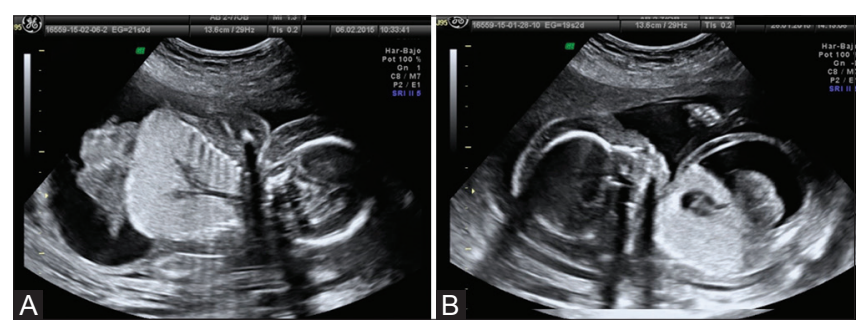

Figure 1 (A-B): Ultrasound examination of CHAOS syndrome: The fetus had bilateral large echogenic lungs and the main bronchi appeared dilated (A). The diaphragm was inverted. The heart was centrally placed and seemed to be compressed by the enlarged lungs (B)

than 35 additional cases have been reported. ${ }^{[5]}$ In case of unrecognized syndrome during the prenatal period, it usually results in stillbirth or death shortly after delivery. ${ }^{[3]}$

Being the syndrome prognosis lethal in case of a CHAOS diagnosis, after the ex utero intra-partum therapy (EXIT)-to-airway strategy, first described in 1998 and 2000, a chance of survival to selected fetus was offered ${ }^{[6,7]}$ The management of isolated cases of CHAOS by fetoscopic decompression of the upper airway has been reported as another successful technique. ${ }^{[8,9]}$ On the other hand, the overall prognosis remains fatal, and long-term medical and surgical challenges for survivors remain considerable even after overcoming fetal intervention. ${ }^{[10]}$

\section{Case History}

A 24-year-old woman, gravida 3, parity 2, was referred for a routine second trimester prenatal ultrasound at 19-week gestational age. The antenatal screening ultrasound at 12 weeks was unremarkable with low risk of chromosomopathy. There was no history of consanguinity. She and her family had unremarkable medical histories.

Ultrasound examination [Video 1] revealed that the fetus had bilateral large echogenic lungs and the main bronchi appeared dilated [Figure 1A]. The diaphragm was inverted. The heart was centrally placed and seemed to be compressed by the enlarged lungs [Figure 1B]. There were severe ascites and anasarca [Figure 1C]. A magnetic resonance imaging (MRI) study was performed which again showed bilateral large and distinctly hyperintense lungs [Video 2]. Significantly, the tracheobronchial tree appeared dilated [Figure 2A]. Inversion of the diaphragm, the typical small compressed fetal heart centrally displaced, and massive ascites were also confirmed [Figure 2B], and no additional anomaly could be found. Based on ultrasound and MRI findings, the diagnosis of CHAOS was confirmed.

The possible unfavorable outcome of the pregnancy was discussed with the couple. She was referred to a genetics specialist and amniocentesis procedure was offered. The
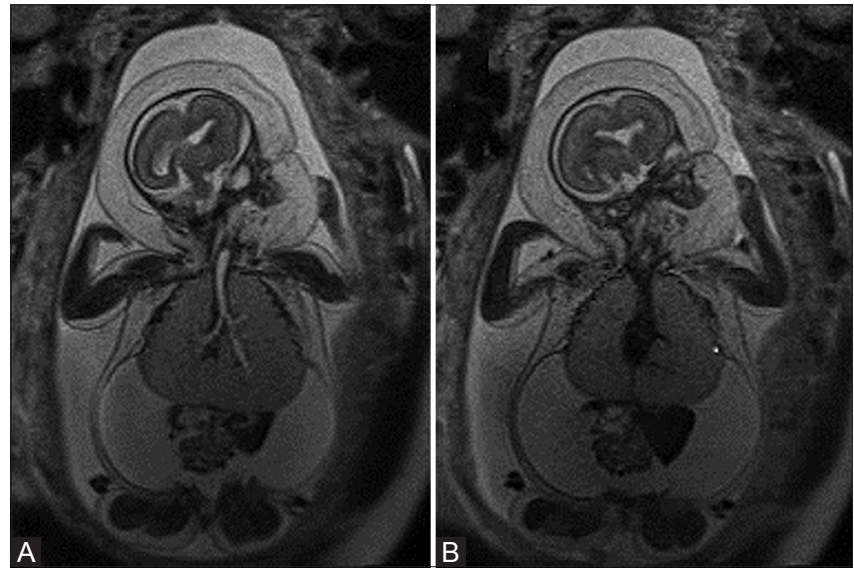

Figure 2 ( $\mathbf{A}$ and $\mathbf{B}$ ): $\mathrm{MRI}$ examination of $\mathrm{CHAOS}$ syndrome: The tracheobronchial tree appeared dilated (A). Inversion of the diaphragm, the typical small compressed fetal heart centrally displaced, and massive ascites were also confirmed (B)

parents refused an amniocentesis and chose to receive expectant management. Any intrauterine or after-delivery treatments were declined. She elected to continue with pregnancy that continued until week 25 , at which time the delivery took place vaginally inducing labor because of maternal risk. The infant died in the following minutes after delivery. A hydropic fetus was born, Apgar 0/0/0 and weight $1475 \mathrm{~g}$. The parents refused an autopsy due to religious reasons.

\section{Discussion}

Postnatal management of newborns with CHAOS is difficult and the prognosis of the affected infants is often poor. Without intervention, the perinatal mortality of CHAOS is $100 \%$. Even though chances of survival may arise after treatments such as fetoscopic procedures and EXIT, CHAOS results in high morbidity-mortality rates. Despite CHAOS has being described in relation to some genetic syndromes and chromosome alterations, the etiology is unknown. Notwithstanding the breakthrough in surgical and management techniques, the prognosis remains very unfavorable.

\section{Declaration of patient consent}

The authors certify that they have obtained all appropriate patient consent forms. In the form the patient(s) has/have given his/her/their consent for his/her/their images and other clinical information to be reported in the journal. The patients understand that their names and initials will not be published and due efforts will be made to conceal their identity, but anonymity cannot be guaranteed.

Financial support and sponsorship

Nil.

\section{Conflicts of interest}

There are no conflicts of interest. 


\section{References}

1. Roybal JL, Liechty KW, Hedrick HL, Bebbington MW, Johnson MP, Coleman BG, et al. Predicting the severity of congenital high airway obstruction syndrome. J Pediatr Surg 2010;45:1633-9.

2. Paladini D, Volpe P. Ultrasound of Congenital Fetal Anomalies: Differential Diagnosis and Prognostic Indicators. $2^{\text {nd }}$ ed. Boca Raton, IL, USA: Taylor and Francis; 2007.

3. Joshi P, Satija L, George R, Chatterjee S, D'Souza J, Raheem A. Congenital high airway obstruction syndrome-Antenatal diagnosis of a rare case of airway obstruction using multimodality imaging. Med J Armed Forces India 2012;68:78-80.

4. Arizawa M, Imai S, Suehara N, Nakayama M. Prenatal diagnosis of laryngeal atresia. Nippon Sanka Fujinka Gakkai Zasshi 1989;41:907-10.

5. Vidaeff AC, Szmuk P, Mastrobattista JM, Rowe TF, Ghelber O. More or less CHAOS: Case report and literature review suggesting the existence of a distinct subtype of congenital high airway obstruction syndrome. Ultrasound Obstet Gynecol 2007;30:114-7.

6. Bui TH, Grunewald C, Frenckner B, Kuylenstierna R, Dahlgren G, Edner A, et al. Successful EXIT (ex utero intrapartum treatment) procedure in a fetus diagnosed prenatally with congenital high-airway obstruction syndrome due to laryngeal atresia. Eur J Pediatr Surg 2000;10:328-33.

7. Crombleholme TM, Sylvester K, Flake AW, Adzick NS Salvage of a fetus with congenital high airway obstruction syndrome by ex utero intrapartum treatment (EXIT) procedure. Fetal Diagn Ther 2000;15:280-2.

8. Kohl T, Hering R, Bauriedel G, Van de Vondel P, Heep A, Keiner S, et al. Fetoscopic and ultrasound-guided decompression of the fetal trachea in a human fetus with Fraser syndrome and congenital high airway obstruction syndrome (CHAOS) from laryngeal atresia. Ultrasound Obstet Gynecol 2006;27:84-8.

9. Kohl T, Van de Vondel P, Stressig R, Wartenberg HC, Heep A, Keiner $\mathrm{S}$, et al. Percutaneous fetoscopic laser decompression of congenital high airway obstruction syndrome (CHAOS) from laryngeal atresia via a single trocar-current technical constraints and potential solutions for future interventions. Fetal Diagn Ther 2009;25:67-71.

10. Saadai P, Jelin EB, Nijagal A, Schecter SC, Hirose S, MacKenzie TC. Long-term outcomes after fetal therapy for congenital high airway obstructive syndrome. J Pediatr Surg 2012;47:1095-100. 